Vysotskii, V. and A.A. Kornilova. The Spatial Structure Of Water And The Problem Of Controlled Low Energy Nuclear Reactions In Water Matrix. in Eleventh International Conference on Condensed Matter Nuclear Science. 2004. Marseille, France.

\title{
THE SPATIAL STRUCTURE OF WATER AND THE PROBLEM OF CONTROLLED LOW ENERGY NUCLEAR REACTIONS IN WATER MATRIX
}

\author{
Vladimir I.Vysotskii $^{1}$, Alla A. Kornilova ${ }^{2}$ \\ ${ }^{1}$ Kiev Shevchenko University, Kiev, Ukraine; \\ ${ }^{2}$ Moscow State University, Moscow, Russia
}

\section{Introduction}

Ordinary water has a number of unique features, among which there are its stable spatial structure and long-term "memory." Numerous experiments confirm the existence of water memory, which is activated under the influence of various physical fields (e.g., magnetic field, mechanical impact, abrupt temperature or pressure change) and may store information about such influence for many hours and days. Such activated water has altered physical and chemical (including biochemical) features. An increasing number of reliable experiments show that the continuous model is inadequate for describing the water structure.

At the first glance, it appears that water, as a specific physico-molecular object, cannot have any long-term memory. It follows from simple estimates.

For a long time, the continuous (quasi crystalline) model of water was the dominant one. Within the framework of this model the spatial structure of potential energy for each one of $\mathrm{H}_{2} \mathrm{O}$ molecules is nearly a periodical three-dimensional system of pits and barriers. This relief is the result of a self-regulating movement of all water molecules, which represents a combination of two independent processes - vibration movement in each one of potential pits and random (fluctuation) leap into a neighboring pit. The average frequency of vibrations in potential pits is approximately the same as the Debay frequency in a solid body (about $\omega_{\mathrm{D}} \approx$ $10^{13} \mathrm{c}^{-1}$ ). The average duration of a leap into a neighboring potential pit is equal $\tau_{0} \approx 10^{-13} \mathrm{c}$. The average time of staying in one pit

$$
\langle\tau\rangle=\tau_{0} \exp (\Delta \mathrm{W} / \mathrm{kT}) \approx 10^{-9}-10^{-10} \mathrm{c}
$$

is determined by the water temperature $\mathrm{T}$ and the energy of activation $\Delta \mathrm{W} \approx 0.2 \mathrm{eV}$ of the diffusion process (the height of the barrier between neighboring pits). Staying within the framework of this model it is easy to reach the conclusion that water memory must be preserved for not much longer than the value $\langle\tau\rangle$, which is by many orders less than given in many experiments.

There can be only two ways out of this logical dead end: either the experiments are not reliable, or the continuous model is incomplete (or wrong).

\section{The problem and the possible mechanism of the water "memory".}

An increasing number of reliable experiments show that the continuous model is inadequate for describing the water structure. More detailed studies have shown that the socalled "clathrate" model is the one closest to reality. In its final form this model was developed by Pauling (1959). On the basis of the Pauling model there is the concept that unification of atoms of oxygen and hydrogen can create spatial flexible tetrahedral frames. 
Formation of a tetrahedral frame was due to the fact that the natural spatial angle between $\mathrm{OH}$-links in a free water molecule $\mathrm{H}_{2} \mathrm{O}$ is equal to $104.5^{\circ}$, which is sufficiently close to the exact value of the tetrahedral angle $108^{\circ}$. In order to achieve an additional angle of $3.5^{\circ}$ in this link an insignificant energy would be required, while the existence of an additional curve would considerably increase rigidity of the crystalline frame (a similar situation occurs, for example, in pure construction material as preliminary stressed concrete).

In the joints of the crystalline frame there are very large (in the scale of a water molecule) micro cavities (microscopic empty spaces) with rigid atomic walls. The main elements of this structure are right polyhedrons linked to each other - dodecahedrons. Such systems are called "clathrate hydrates". The entire frame is held together by hydrogen links. They fasten together a system of pentagonal dodecahedronic polyhedrons from ions of oxygen and hydrogen, which form the walls of the micro cavities. Each one of the polyhedrons may be characterized by an inscribed sphere with a radius of about $\mathrm{R}_{\mathrm{c}} \approx 2.6 \mathrm{~A}$. All polyhedrons have 12 pentagonal facets, 30 edges connecting these facets and 20 vertexes with 3 edges converging in each one of them. At the vertexes of these polyhedrons there are 20 molecules of water, $\mathrm{H}_{2} \mathrm{O}$, each one having three hydrogen links. Any 3 polyhedrons may be unified into stable associates containing 57 molecules of water. Out of these 57 molecules, 17 have fully saturated hydrogen links and they form a tetrahedral hydrophobic central frame, while in 4 dodecahedrons there are 10 centers of formation of hydrogen links $(\mathrm{O}-\mathrm{H}$ or $\mathrm{O})$ located on the surface of each one. The space structure of the system of clathrate hydrates in water is presented in Fig. 1.

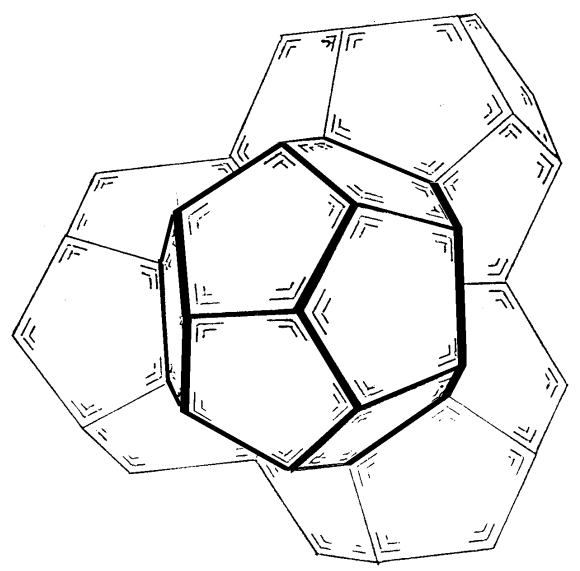

Fig. 1. The system of clathrate hydrates in water

Beyond this frame are quasi-free molecules of "regular" isotropic water, the features and the structure of which approximately match the continuous model. Micro cavities are linked to the outer space by windows with diameter of about $2.5 \mathrm{~A}$, which is slightly less than diameter of a water molecule $\left(2 \mathrm{R}_{\mathrm{W}} \approx 2.76 \mathrm{~A}\right)$. As a result, each of the micro cavities is separated from "external" amorphous quasi-free water by a circular potential barrier with width about 0.13-0.15 A bounding each window. The relative quantity of molecules of "frame" water at room temperature is $20-30 \%$, increasing at lower temperatures. The volume of these micro cavities may be large enough to hold one molecule of $\mathrm{H}_{2} \mathrm{O}, \mathrm{CH}_{4}, \mathrm{O}_{2}$ or $\mathrm{N}_{2}$.

Due to the presence of a strong and symmetrical electrostatic field (symmetrical relative the center of micro cavities), there is a certain ban on the formation of hydrogen links of water molecules with the walls inside the micro cavities. In this case there is such a non-trivial phenomenon as repulsion of free water molecules from the walls of the frame also consisting of water molecules. (In other words, water molecules in the volume of water become hydrophobic)! The average density of a clathrate frame (without filling it up with water 
molecules) equals $0.80 \mathrm{~g} / \mathrm{cm}^{3}$, i.e. the micro cavities occupy $20 \%$ of the full volume of a structured water frame. If the micro cavities are saturated with molecules of water the density of water would be close to $1 \mathrm{~g} / \mathrm{cm}^{3}$.

We shall demonstrate how the presence of a clathrate frame of water may lead to formation of long-term memory in it, and to recording and using information [1].

Let us examine initial water in the state of thermodynamic balance with a certain temperature T. This condition is distinguished by maximal entropy. Such water is obtained by long boiling and slow cooling down, or by a very long-term storage. In this case the number of micro cavities within the system of clathrate hydrates filled with water matches the Bolzman distribution accounting for statistical weights of $\mathrm{H}_{2} \mathrm{O}$ molecules' condition in micro cavities and in amorphous water. It would be balanced or regular water.

At the temperature of $4^{\circ} \mathrm{C} 18 \%$ of all micro cavities are filled with water. At normal body temperature $\left(36.6^{\circ} \mathrm{C}\right) 38 \%$ of micro cavities are filled, while at $55^{\circ} \mathrm{C}$ about $50 \%$ of micro cavities will be occupied by $\mathrm{H}_{2} \mathrm{O}$ molecules.

This pattern of distribution is related to several factors:

1. The Bolzman distribution at a given temperature;

2. The repetition factor of degeneration of the initial and final state of an $\mathrm{H}_{2} \mathrm{O}$ molecule in amorphous water near an entry window into the volume of a micro cavity and inside it;

3. The ratio of the volume of all amorphous water and the volume of a clathrate frame.

With changing temperature all three values change, which makes exact calculations of the dynamics of occupation of micro cavities more difficult. However, it is obvious that in the volume of clathrate micro cavities the energy of molecular links is close to zero (due to hydrophobic nature of interaction with the walls), while condition of an $\mathrm{H}_{2} \mathrm{O}$ molecule in the volume of quasi-amorphous water is determined by the depth of a potential pit, dictated by links with other water molecules. The depth of that pit corresponds to the energy of activation at diffusion $\Delta \mathrm{W} \approx 0.2 \mathrm{eV}$, which, in effect, reduces the level of energy of an $\mathrm{H}_{2} \mathrm{O}$ molecule with respect to condition of the same molecule in the clathrate frame by the value of $\Delta \mathrm{W}$. For these reasons, it becomes clear that the necessary activation energy to entering a micro cavity $\Delta \mathrm{E}_{\mathrm{M}}$, and exit from it $\Delta \mathrm{E}_{\mathrm{M}}-\Delta \mathrm{E}$, would be different (Fig. 2).

Based on this, the time that an "extra" molecule of water would stay in a micro cavity and the time an "extra" vacancy in an empty micro cavity exists would also be different.

With a violation of thermodynamic balance a redistribution of $\mathrm{H}_{2} \mathrm{O}$ molecules between amorphous water and micro cavities takes place until a new balanced state is achieved. We shall demonstrate that a spontaneous transfer between these states is substantially inhibited due to the very small probability of tunnel penetration of $\mathrm{H}_{2} \mathrm{O}$ molecules through "narrow" windows, and the time of existence of each of these conditions turns out to be very long. We shall determine the time of relaxation in such a redistribution.

Such relaxation corresponds to a transfer of water molecules in two possible directions: a) from the state of amorphous water into the volume of a micro cavity (if the initial number of water molecules in micro cavities was smaller than the value of a variable determined by the Bolzman distribution, which may happen in case of a rapid heating of water); b) from the state of "excess" water in micro cavities to amorphous water (if the number of water molecules in micro cavities exceeded the value for balanced state, which, for example, corresponds to the case of a rapid cooling down of water).

The process of relaxation of each of these states depends on thermodynamic probability

$$
\mathrm{W}=\exp \left(-\Delta \mathrm{E}_{\mathrm{M}} / \mathrm{k}_{\mathrm{B}} \mathrm{T}\right)
$$

that one of the water molecules after its interaction with other molecules will receive energy $\Delta \mathrm{E}_{\mathrm{M}}$ sufficient for short-term deformation (i.e. work on increasing energy of interaction 
between a proton and an ion of oxygen), which would be enough for a reduction of the water molecule's size to the dimensions of a window of a micro cavity and, correspondingly, penetration of that molecule inside the micro cavity.

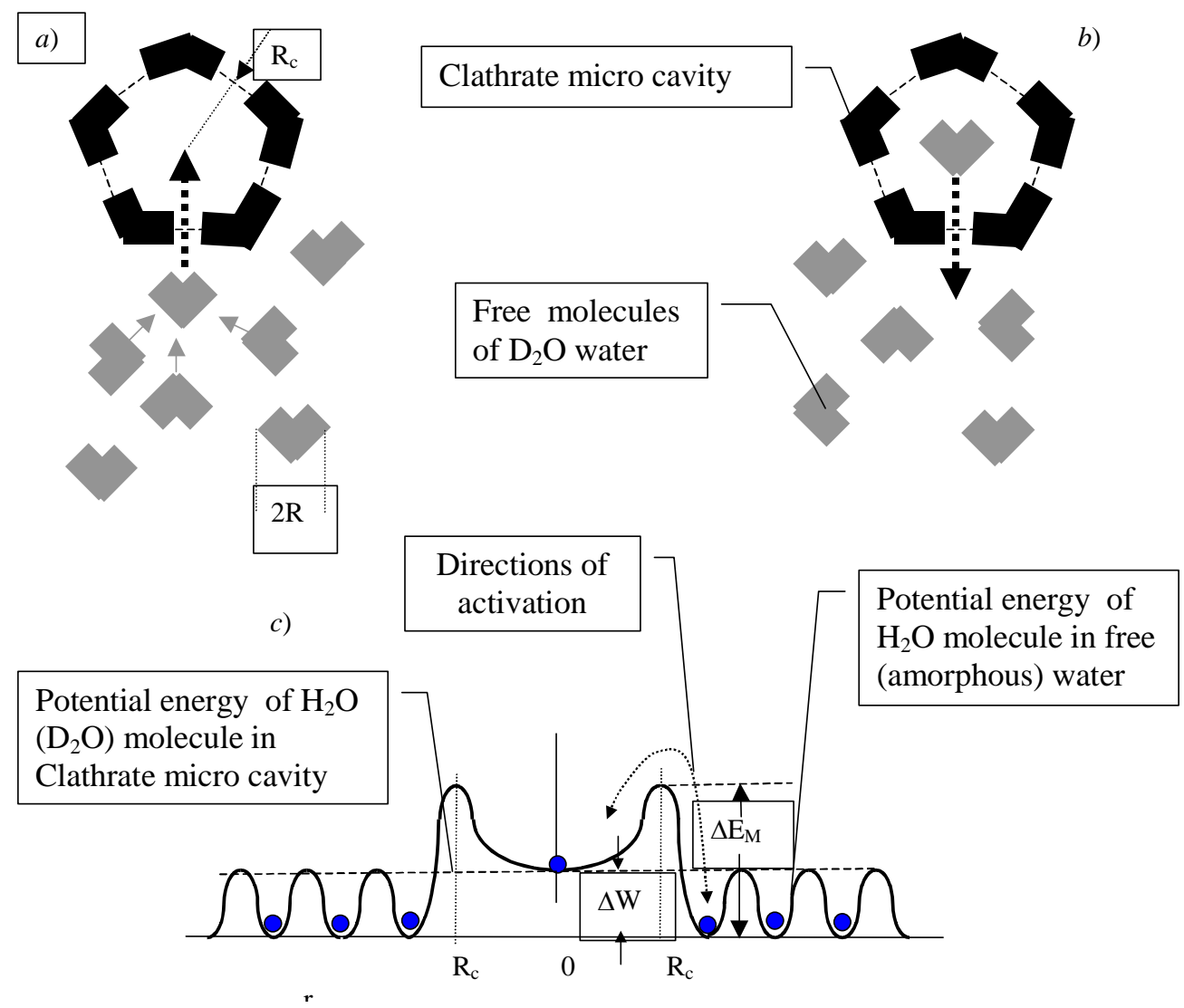

Fig. 2. Process of thermally stimulated activation $a$ ) and deactivation $b$ ) of micro cavities of a clathrate water frame at increasing and decreasing temperatures; $c$ ) structure of potential energy of molecules of amorphous and linked water in the volume of a clathrate micro cavity and around its boundaries

Since the frequency of collisions of a water molecule with the surface of any structured object in water is equal to the frequency of vibrations of molecules around a local position of balance $\omega_{\mathrm{D}} \approx 1 / \tau_{0} \approx 10^{13} \mathrm{~s}$, the total probability of capturing a molecule in a unit of time into an empty micro cavity is equal to $\mathrm{F}=\mathrm{W} / \tau_{0}$. From this expression we can determine the average lifetime of an unbalanced (empty) state of a single micro cavity in the volume of a spatial tetrahedral water frame (time of relaxation of a vacant spot in a micro cavity)

$$
\mathrm{T}_{1 \mathrm{~W}}=1 / \mathrm{F}_{1}=\tau_{0} \exp \left(\Delta \mathrm{E}_{\mathrm{M}} / \mathrm{k}_{\mathrm{B}} \mathrm{T}\right)
$$

Plainly, that time will determine the duration of water memory before the micro cavity is filled (erased), for example, when the water is heated.

It is possible to calculate the value of $\Delta \mathrm{E}_{\mathrm{M}}$, characterizing that process.

The fluctuational movement of a proton in an $\mathrm{H}_{2} \mathrm{O}$ molecule in a direction perpendicular to the line of link $\mathrm{OH}$ constitutes that of a harmonic oscillator.

The potential energy representing shifting of an ion of hydrogen by the value $r$ with respect to the point of balance may be expressed in the form of energy of a harmonic oscillator 


$$
V(r)=M_{H} \omega_{H}^{2} r^{2} / 2
$$

Here $\mathrm{M}_{\mathrm{H}}-$ mass of an atom of hydrogen, $\omega_{\mathrm{H}} \approx 3 * 10^{14} \mathrm{c}^{-1}-$ frequency of normal vibrations of a proton in a molecule of water in a direction perpendicular to the line of the $\mathrm{OH}$ link [1].

Taking into account that the angle between the lines linking each of the protons with the nucleus of oxygen equals $2 \alpha \approx 104.5^{\circ}$, we find that energy required for deformation of the outer dimensions of a water molecule by the value of $\Delta \mathrm{R} \approx 0.26 \mathrm{~A}$, sufficient for putting a molecule inside a micro cavity would be

$$
\mathrm{V}(\Delta \mathrm{R})=\mathrm{M}_{\mathrm{H}} \omega_{\mathrm{H}}^{2} \Delta \mathrm{R}^{2} / 2 \cos ^{2} \alpha \approx 1.1 \mathrm{eV}
$$

This variable represents energy threshold $\Delta \mathrm{E}_{\mathrm{M}}=\mathrm{V}(\Delta \mathrm{R})$ determining the process of water relaxation. This threshold greatly exceeds the thermal energy of water molecules, which is equal to $\mathrm{k}_{\mathrm{B}} \mathrm{T} \approx 0.025 \mathrm{eV}$ at room temperature. It can be seen, then, that time of relaxation $\mathrm{T}_{1 \mathrm{~W}}$ is strongly correlated with the threshold value for energy of deformation of a water molecule $\Delta \mathrm{E}_{\mathrm{M}}$ and its temperature $\mathrm{T}$. A big value of $\Delta \mathrm{E}_{\mathrm{M}}$ leads to a small probability of overcoming the barrier in the area of an entrance window to a micro cavity. In the result, the probability of spontaneous deactivation of water is very small, which means a very long period of storing information.

Let us make some quantitative estimates. At the temperature of water $\mathrm{T}=293 \mathrm{~K}\left(20^{\circ} \mathrm{C}\right)$ the time of relaxation (the duration of "water memory") is equal to $\mathrm{T}_{1 \mathrm{~W}} \approx 10$ days. With higher water temperatures time of relaxation decreases sharply, and it increases with cooling down (see Table 1).

For an alternative direction of relaxation (transferring a single molecule of water from the volume of a micro cavity into the volume of amorphous water), the time of relaxation $T_{2 W}$ is also determined by an expression similar to (3), where energy of activation is different $\left(\Delta \mathrm{E}_{\mathrm{M}}\right.$ $\Delta \mathrm{E} \approx 0.9 \mathrm{eV}$ instead of $\Delta \mathrm{E}_{\mathrm{M}} \approx 1.1 \mathrm{eV}$ ). Moreover, it is necessary to remember that because the inner dimension of a micro cavity is considerably bigger than the size of a potential pit for each molecule in the volume of quasi-amorphous water, the actual frequency of collisions of a water molecule with the walls inside a micro cavity $\omega_{\mathrm{D}} \approx 1 / \tau_{0}$ will be lower, while period $\tau_{0}$ will be, respectively, larger than in the volume of water. The results of calculation of time of relaxation $\mathrm{T}_{2 \mathrm{~W}}$ during a reverse transferring of molecules $\mathrm{H}_{2} \mathrm{O}$ from a volume of a micro cavity into quasi-amorphous water are also presented in Table 1.

Table 1. Times of relaxation of water (duration of "water memory").

\begin{tabular}{|l|l|l|l|l|l|l|l|l|l|l|}
\hline $\mathrm{T}^{\mathrm{o}} \mathrm{C}$ & 1 & 10 & 20 & 30 & 36.6 & 40 & 50 & 60 & 70 & 90 \\
\hline $\mathrm{T}_{1 \mathrm{~W}}$ & $\begin{array}{l}300 \\
\text { days }\end{array}$ & $\begin{array}{l}49 \\
\text { days }\end{array}$ & $\begin{array}{l}10 \\
\text { days }\end{array}$ & $\begin{array}{l}58 \\
\text { hours }\end{array}$ & $\begin{array}{l}24 \\
\text { hours }\end{array}$ & $\begin{array}{l}15 \\
\text { hours }\end{array}$ & $\begin{array}{l}4.4 \\
\text { hours }\end{array}$ & $\begin{array}{l}1.3 \\
\text { hours }\end{array}$ & $\begin{array}{l}27 \\
\text { min }\end{array}$ & $\begin{array}{l}3 \\
\text { min }\end{array}$ \\
\hline $\mathrm{T}_{2 \mathrm{~W}}$ & $\begin{array}{l}30 \\
\text { min }\end{array}$ & $\begin{array}{l}14 \\
\text { min }\end{array}$ & $\begin{array}{l}4 \\
\text { min }\end{array}$ & $\begin{array}{l}1.5 \\
\text { min }\end{array}$ & $\begin{array}{l}45 \\
\text { sec }\end{array}$ & $\begin{array}{l}30 \\
\text { sec }\end{array}$ & $\begin{array}{l}12 \\
\text { sec }\end{array}$ & $\begin{array}{l}4 \\
\text { sec }\end{array}$ & $\begin{array}{l}1.5 \\
\text { sec }\end{array}$ & $\begin{array}{l}0.3 \\
\text { sec }\end{array}$ \\
\hline
\end{tabular}

It should be noted that in order to calculate values of $\mathrm{T}_{1 \mathrm{~W}}$ and $\mathrm{T}_{2 \mathrm{~W}}$ we need to know the exact values for the energy of activation and the height of a potential barrier regulating the entrance into volume of micro cavities of the clathrate frame. These parameters have been 
determined by us from model calculations. Respective values of $\mathrm{T}_{1 \mathrm{w}}$ and $\mathrm{T}_{2 \mathrm{~W}}$ may differ considerably in specification of these parameters.

The obtained values of $\mathrm{T}_{1 \mathrm{~W}}$ and $\mathrm{T}_{2 \mathrm{~W}}$ represent water relaxation from an unstable state to a stable one, corresponding to a specific temperature.

If we examine this process from the point of view of information theory, the generation of an unbalanced distribution may be considered a process of recording information in a volume of water. We shall call such water activated.

A very long time of relaxation $\mathrm{T}_{1 \mathrm{w}}$ allows us to assume that water is a two-level (or rather double-zone) bi-stable system with a long lifetime in each of those states. Such systems allow the recording and holding of information (in the form of ratio of occupied and vacant micro cavities) as well as using that information effectively by altering the properties of water at time of transferring of a large number of $\mathrm{H}_{2} \mathrm{O}$ molecules and other atoms dissolved in water, as well as molecules and ions from the state of amorphous water into the volume of linked micro cavities, or the other way around (Fig. 2).

Although the time of reverse relaxation $\mathrm{T}_{2 \mathrm{~W}}$ at the exit of water molecules from the volume of micro cavities turns out to be considerably lower than time of direct relaxation at the entrance to these micro cavities, in any case, it is many orders of magnitude greater than the typical time of relaxation $(1)\langle\tau\rangle \approx 10^{-9}-10^{-10}$ s due to fluctuations of the hydrogen link in the volume of amorphous water.

It may also be noted that the process of activation of water may be conducted not only during its heating or cooling, but also through the effects of magnetic fields or ultrasound. Such periodic coherent influences can stimulate formation of quasi-stable clusters, each one of which unites several reciprocally arranged cluster frames. In such a system, the behavior of isolated water molecules in periodically situated micro cavities is similar to movement of hydrogen in palladium, where a very high saturation of lattice is achieved. Periodic influences can also affect the parameters of the clathrate frame of water, altering, for example, the transparency of the potential barrier in windows of micro cavities. (This is the problem with tunneling a molecule $\mathrm{H}_{2} \mathrm{O}$ through a non-stationary barrier.) Moreover, a strong periodic magnetic field can stimulate transfers between energy levels, which characterize the state of $\mathrm{H}_{2} \mathrm{O}$ molecules in micro cavities and amorphous water (for example, due to multi-photon nonlinear processes during interaction with magnetic moments), which causes uneven populating of micro cavities by water molecules and constitutes activation of water.

\section{The mechanism of time-dependent self-organization of Coulomb-free nuclear reactions in optimal potential wells in dynamic systems}

The same clathrate space structure applies to heavy water $\left(\mathrm{D}_{2} \mathrm{O}\right)$. The potential energy of the $\mathrm{D}$ atom in the central part of the cavity with "water walls" is close to parabolic. The possible mechanism of nonbarier nuclear reaction in optimal potential holes was described in [2].

In such well the spectrum of quantum energy levels is equidistant and

$$
E_{n}=\hbar \omega_{0}(n+3 / 2), n=0,1,2, \ldots
$$

Let one atom $X$ be in the center of such a well. When other atom $Y$ gets into the well (e.g. due to diffusion), a complex $\mathrm{X}+\mathrm{Y}$ appears in the well. In the free space (e.g. for a well with the size $\mathrm{R} \rightarrow \infty$ ) this complex would correspond to quasimolecule (XY).

In the quantum system the situation is more complicated, because in such a system energy of total X - Y interaction V(r) is a sign-variable distance function (e.g [2]) which is important for the calculation of diagonal matrix elements 


$$
\left.\mathrm{V}_{\mathrm{nn}}=\int_{0}^{\mathrm{R}} 4 \pi\left|\psi_{\mathrm{n}}(\mathrm{r})\right|^{2} \mathrm{~V}(\mathrm{r}) \mathrm{r}^{2} \mathrm{dr}, a=\hbar^{2} / \mathrm{me}^{2}\right)
$$

The main question is: Can this energy be a small perturbation and not influence the character of the atoms or nuclei movement and interaction in quantum system? For that purpose, the diagonal elements of interaction energy matrix should be small (a) and probability of interlayer transition because of this interaction should also be small (b).

The probability of interlayer transition in the parabolic potential well becomes equal to zero automatically at the optimal moments of time $\tau=2 \mathrm{~s} \pi / \omega_{0}, \mathrm{~s}=1,2,3 \ldots$, when the frequencies of all possible interlayer transitions $\omega_{\mathrm{nk}}=(\mathrm{n}-\mathrm{k}) \omega_{0}$ will correspond to zero spectral density of perturbation energy (see Fig. 2)

$$
|V(\omega)|^{2}=\left|\int_{0}^{\tau} \int V_{n k}(t) \exp (i \omega t) d t\right|^{2}=V_{n k}[\sin (\omega \tau / 2) /(\omega / 2)]^{2}
$$

Here atoms or nuclei interaction completely disappears and wave function of atoms is determined only by quantum potential field (well) properties.

To zero the diagonal matrix elements of interaction energy (which is a sign-variable function of inter-nucleus distance) $\mathrm{V}_{\mathrm{nn}}$ it is essential that the size of the hole $\mathrm{R}$ be optimal. For the typical atom in the $\mathrm{X}-\mathrm{Y}$ system this optimal size is $\mathrm{R} \approx 4 \mathrm{~A}$.

If all the above-mentioned conditions (a) and (b) are met, the independent from first atom $\mathrm{X}$ quantizing of the second atom $\mathrm{Y}$ in the optimal well takes place. In this case the wave

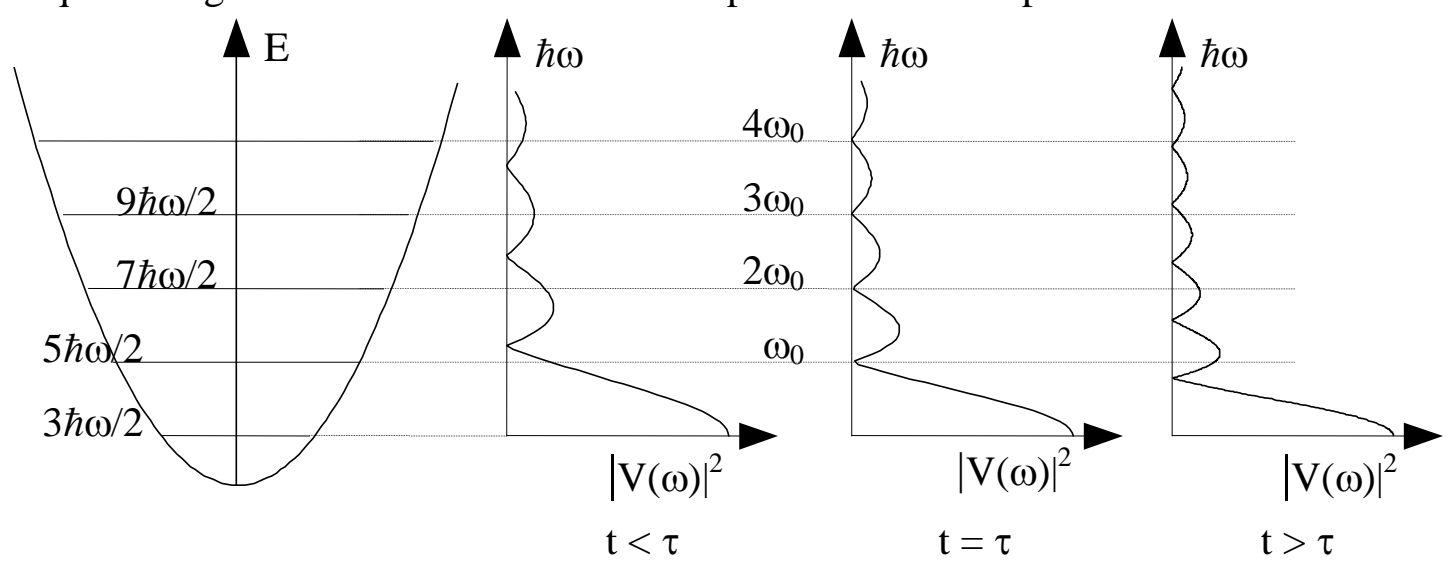

Fig. 5. Correlation between energy spectrum of quantum levels $E_{n}$ and spectral density $|V(\omega)|^{2}$ of perturbation energy $V(t)$ for $t<\tau, t=\tau, t>\tau$.

function of this atom $\psi_{\mathrm{n}}(0)$ in all even states will be different from zero in the center of the well, where the first atom $\mathrm{X}$ is located.

This leads to a high probability $\lambda=\mathrm{C}\left|\psi_{\mathrm{n}}(0)\right|^{2}$ of nuclear fusion.

In such a well, the spectrum of quantum levels of $D$ atom is equidistant. In such a compressed, isolated system the energy of dd Coulomb interaction of two D atoms is a small amendment and does not influence the character of atoms or nuclei movement and interaction (including dd-fusion) [1]. Each such micro cavity resembles a nonthreshold micro reactor in water volume. 


\section{References}

[1]. Vysotskii V.I., Kornilova A.A. Physical foundation of long-time water memory, Moscow University Physics Bulletin, v.59, no.3, p.58, 2004

[2]. Vysotskii V.I., Kornilova A.A. Nuclear fusion and transmutation of isotopes in biological systems, Moscow, "MIR" Publishing House, 2003, 302 pages. 